\title{
Candida albicans Mycofilms Support Staphylococcus aureus Colonization and Enhances Miconazole Resistance in Dual-Species Interactions
}

\author{
Ryan Kean 1,2, Ranjith Rajendran', Jennifer Haggarty³, Eleanor M. Townsend ${ }^{1,2}$, \\ Bryn Short'1,4, Karl E. Burgess ${ }^{3}$, Sue Lang ${ }^{4}$, Owain Millington ${ }^{5}$, William G. Mackay ${ }^{2}$, \\ Craig Williams ${ }^{2}$ and Gordon Ramage ${ }^{1 *}$
}

\begin{abstract}
${ }^{1}$ Oral Sciences Research Group, Glasgow Dental School - School of Medicine, Dentistry and Nursing, College of Medical, Veterinary and Life Sciences, University of Glasgow, Glasgow, UK, ${ }^{2}$ Institute of Healthcare Policy and Practise - Institute of Healthcare Associated Infection, University of the West of Scotland, Paisley, UK, ${ }^{3}$ Glasgow Polyomics, University of Glasgow, Glasgow, UK, ${ }^{4}$ Department of Life Sciences, School of Health and Life Sciences, Glasgow Caledonian University, Glasgow, UK, ${ }^{5}$ Strathclyde Institute of Pharmacy and Biomedical Sciences, University of Strathclyde, Glasgow, UK
\end{abstract}

\section{OPEN ACCESS}

Edited by:

Luis R. Martinez,

New York Institute of Technology, USA

Reviewed by:

Patrick Van Dijck,

KU Leuven, Belgium

Catherine Ann Wakeman,

Texas Tech University, USA

*Correspondence:

Gordon Ramage

gordon.ramage@glasgow.ac.uk

Specialty section: This article was submitted to Fungi and Their Interactions,

a section of the journal

Frontiers in Microbiology

Received: 25 November 2016 Accepted: 07 February 2017

Published: 23 February 2017

Citation:

Kean $R$, Rajendran $R$, Haggarty $J$, Townsend EM, Short B, Burgess KE,

Lang S, Millington O, Mackay WG,

Williams C and Ramage G (2017) Candida albicans Mycofilms Support

Staphylococcus aureus Colonization and Enhances Miconazole Resistance

in Dual-Species Interactions.

Front. Microbiol. 8:258.

doi: 10.3389/fmicb.2017.00258
Polymicrobial inter-kingdom biofilm infections represent a clinical management conundrum. The presence of co-isolation of bacteria and fungi complicates the ability to routinely administer single antimicrobial regimens, and synergy between the microorganisms influences infection severity. We therefore investigated the nosocomial pathogens Staphylococcus aureus and Candida albicans with respect to antimicrobial intervention. We characterized the interaction using biofilm assays and evaluated the effect of miconazole treatment using in vitro and in vivo assays. Finally, we assessed the impact of biofilm extracellular matrix (ECM) on these interactions. Data indicated that the C. albicans mycofilms supported adhesion and colonization by S. aureus through close interactions with hyphal elements, significantly increasing $S$. aureus biofilm formation throughout biofilm maturation. Miconazole sensitivity was shown to be reduced in both mono- and dual-species biofilms compared to planktonic cells. Within a threedimensional biofilm model sensitivity was also hindered. Galleria mellonella survival analysis showed both enhanced pathogenicity of the dual-species infection, which was concomitantly desensitized to miconazole treatment. Analysis of the ECM revealed the importance of extracellular DNA, which supported the adhesion of $S$. aureus and the development of the dual-species biofilm structures. Collectively, these data highlight the clinical importance of dual-species inter-kingdom biofilm infections, though also provides translational opportunities to manage them more effectively.

Keywords: Candida albicans, Staphylococcus aureus, miconazole, biofilm, extracellular DNA

\section{INTRODUCTION}

Despite their clinical significance, polymicrobial biofilm infections continue to be a widely understudied health problem. Advancement in biofilm research has highlighted that these cellular communities are rarely composed of a single-species consortia, but instead exist as complex, diverse, and heterogeneous structures (Peleg et al., 2010). By actively participating within these 
environments different micro-organisms can interact at a mechanistic level through direct and indirect exchanges of both a physical and chemical nature. This can ultimately influence disease severity by promoting intensified pathogenic phenotypes (Peters et al., 2010), including increased recalcitrance to both host defenses and antimicrobial therapies (Kart et al., 2014). Although belonging to distinct phylogenetic kingdoms, the dimorphic fungus Candida albicans and the bacterial pathogen Staphylococcus aureus possess the ability to habitually co-exist as complex polymicrobial biofilms within the human host, and are therefore key exemplars of polymicrobiality.

Staphylococcus aureus and C. albicans are defined as the second and fourth most commonly cultured organisms from bloodstream infections within the USA (Wisplinghoff et al., 2004). An estimated $27 \%$ of candidaemia infections are polymicrobial, with $S$. aureus representing the third most frequently co-isolated organism (Klotz et al., 2007). Duality of infection is also frequently observed in co-infections of burn wounds and cystic fibrosis, as well from the surface of indwelling medical devices such as dentures, prosthetic joints and implants, and more commonly catheters (Valenza et al., 2008; Peters et al., 2012a). Mechanistically, there is evidence to suggest that this interaction is multifaceted, intricately linked to biofilm-phased development. Initial attachment of $S$. aureus to C. albicans hyphae appears to be mediated by $C$. albicans agglutinin like sequence 3 protein (Als3p; Peters et al., 2012b). As the biofilm develops, quorum sensing (QS) molecules have been shown to exhibit both synergistic and antagonistic effects, with either organism secreting reciprocal QS molecules to positively and negatively influence biofilm growth (Jabra-Rizk et al., 2006; Lin et al., 2013). Structurally, the extracellular matrix (ECM) of $C$. albicans has been shown to advantageously support these dual-species biofilms through prevention of diffusion of vancomycin to access $S$. aureus, with the bacteria potentially encasing itself within this polymeric material (Harriott and Noverr, 2009). Furthermore, it has now been shown that the fungal specific matrix component $\beta$-1,3-glucan promotes this vancomycin resistance (Kong et al., 2016). However, how these biofilms respond to antifungal agents remains undefined.

Candida albicans biofilms are notoriously resistant to antifungal agents, displaying up to 1000-fold increases in resistance to azoles compared to their planktonic counterparts (Ramage et al., 2001). Triazoles, such as fluconazole, represent the first-line option for C. albicans infections, though are often ineffectual against C. albicans biofilms (Sherry et al., 2014; Rajendran et al., 2016b). However, unlike these more commonly used triazoles, the imidazole miconazole appears to display fungicidal activity against C. albicans biofilms in vitro (Vandenbosch et al., 2010). This same agent has also been shown to display antibacterial activity against a range of Grampositive bacteria, including S. aureus (Nobre et al., 2010). We therefore sought to evaluate the in vitro and in vivo efficacy of miconazole against $C$. albicans and $S$. aureus dual-species biofilms, and aimed to understand the contribution of the ECM in this interaction. Here we report that C. albicans mycofilms actively enhance $S$. aureus colonization and negatively impacts miconazole sensitivity.

\section{MATERIALS AND METHODS}

\section{Microbial Growth and Standardization}

Candida albicans SC5314 strain and the biofilm defective Staphylococcus aureus Newman strain were used throughout the duration of this study (Duthie and Lorenz, 1952; Fonzi and Irwin, 1993; Beenken et al., 2003). C. albicans SC5314 was grown and maintained on Sabouraud's dextrose agar (Oxoid, Hampshire, $\mathrm{UK}$ ) and incubated at $30^{\circ} \mathrm{C}$ for $48 \mathrm{~h}$. Yeast cells were propagated by inoculating yeast peptone dextrose (YPD) media (SigmaAldrich, Dorset, UK) with a loopful of colonies and incubated at $30^{\circ} \mathrm{C}$ at $200 \mathrm{rpm}$ in an orbital shaker overnight. After growth, cells were pelleted by centrifugation and washed in phosphate buffered saline [PBS (Sigma-Aldrich, Dorset, UK)] before being standardized in selected media at assay specific concentration requirements after cells were counted using a haemocytometer. The $S$. aureus Newman strain was cultured and maintained on Luria Bertani agar at $37^{\circ} \mathrm{C}$ for $48 \mathrm{~h}$. S. aureus cells were propagated overnight in Luria Bertani broth at $37^{\circ} \mathrm{C}$ before being washed by centrifugation in PBS. Following cell washing, they were standardized in selected media to assay specific concentration requirements after their density was determined using a colorimeter.

\section{Development of Dual-Species Biofilm Model}

Both $C$. albicans and $S$. aureus were standardized to $1 \times 10^{6}$ cells $/ \mathrm{mL}$ in $50 \% \mathrm{v} / \mathrm{v}$ foetal bovine serum (FBS) diluted in sterile water. Biofilms were formed at $37^{\circ} \mathrm{C}$ over 1.5 (90 $\left.\mathrm{min}\right), 6$ and $24 \mathrm{~h}$ by adding both organisms $1: 1$ to either microtiter plates (Corning, NY, USA) or $13 \mathrm{~mm}$ diameter Thermanox ${ }^{\mathrm{TM}}$ coverslips (Fisher Scientific, Loughborough, UK), depending on the assay function. Mono-species biofilms and appropriate media controls were run in parallel for each experimental condition assessed. Following incubation media was discarded and biofilms were washed with PBS to remove any non-adherent cells. The biomass of each biofilm was quantified by the crystal violet (CV) assay described previously (Sherry et al., 2014). The $\mathrm{CV}$ absorbance was then measured spectrophotometrically at a wavelength of $570 \mathrm{~nm}$ using a microtiter plate reader (FLUOStar Omega, BMG Labtech, Aylesbury, UK).

\section{Biofilm Composition and Viability Analysis}

Biofilm composition was enumerated using live/dead PCR, a technique also able to differentiate between total and live cells using methodologies previously described by our group (Townsend et al., 2016). Biofilms were grown as above on Thermanox $^{\mathrm{TM}}$ coverslips (Fisher Scientific, Loughborough, UK), washed in PBS, and sonicated in $1 \mathrm{~mL}$ of PBS at $35 \mathrm{kHz}$ in an ultrasonic water-bath (Fisher Scientific, Loughborough, UK) for $15 \mathrm{~min}$ to remove the biofilm. Next, $50 \mu \mathrm{M}$ of propidium monoazide [PMA (Cambridge Bioscience, Cambridge, UK)] was added to the sample and incubated for $10 \mathrm{~min}$ in the dark to allow uptake of the dye. PMA-free controls were also included to determine total cell number. All samples were then exposed 
to a $650 \mathrm{~W}$ halogen light for $5 \mathrm{~min}$ before DNA was extracted using the QIAamp DNA mini kit, as per manufacturer's protocol (Qiagen, Crawley, UK). Real-time quantitative PCR (qPCR) was then used to determine the live and total cell number within each biofilm. Briefly, $1 \mu \mathrm{L}$ of extracted DNA was added to a PCR mastermix containing Fast $\mathrm{SYBR}^{\circledR}$ Green Master Mix, $10 \mu \mathrm{M}$ species-specific forward and reverse primers (Table 1), and RNase free water. qPCR was then carried out using the StepOne plus real time PCR machine (Life Technologies, Paisley, UK) using the following thermal profile: $50^{\circ} \mathrm{C}$ for $2 \mathrm{~min}, 95^{\circ} \mathrm{C}$ for $2 \mathrm{~min}$, followed by 40 cycles of $95^{\circ} \mathrm{C}$ for $3 \mathrm{~s}$ and $60^{\circ} \mathrm{C}$ for $30 \mathrm{~s}$. Colony forming equivalents (CFE) were calculated compared to a standard curve of serially diluted DNA of each species as previously described (O’Donnell et al., 2016).

\section{Dual-Species Biofilm Visualization}

Mono- and dual-species biofilms were stained with $5 \mu \mathrm{M}$ calcofluor white (Invitrogen, Paisley, UK) and $20 \mu \mathrm{M}$ SYTO9 ${ }^{\circledR}$ (Sigma-Aldrich, Dorset, UK) was used to stain both fungal and bacterial cells. Biofilms were then imaged using a confocal laser scanning microscopy (CLSM) microscope (Leica SP5 laser scanning confocal microscope) at an excitation and emission wavelength of 350 and $400 \mathrm{~nm}$ for calcofluor white and 485 and $500 \mathrm{~nm}$ for $\mathrm{SYTO}^{\circledR}$. Images were then processed and analyzed using Volocity 3D Image Analysis Software (Perkin Elmer). In addition, biofilms were also imaged using scanning electron microscopy (SEM). After biofilm development, biofilms were fixed using 2\% para-formaldehyde, $2 \%$ glutaraldehyde, $0.15 \mathrm{M}$ sodium cacodylate, and $0.15 \% \mathrm{w} / \mathrm{v}$ alcian blue and processed for SEM, as previously described (Erlandsen et al., 2004). Samples were then sputter coated in gold before being imaged using a JEOL JSM-6400 scanning electron microscope.

\section{In vitro Biofilm Susceptibility Testing}

Simple mono- and dual-species biofilms were grown in microtiter wells for $24 \mathrm{~h}$ before being challenged for a subsequent $24 \mathrm{~h}$ with serially twofold diluted concentrations of miconazole (SigmaAldrich, Dorset, UK), ranging from 320 to $0.63 \mathrm{mg} / \mathrm{L}$ using standardized biofilm testing methodology to determine the sessile minimum inhibitory concentration (SMIC; Sherry et al., 2014). Planktonic MIC (PMIC) testing was also performed on single- and dual-species inoculum using standard CLSI broth microdilution testing (CLSI, 2012). In addition, these biofilms were also treated with miconazole \pm DNase I $[130 \mathrm{mg} / \mathrm{L}$ (SigmaAldrich, Dorset, UK)], lyticase, and chitinase [50 mg/L (SigmaAldrich, Dorset, UK)]. Following treatment the proportional viability was calculated in each test condition compared to an untreated control using the XTT metabolic reduction assay (Pierce et al., 2008).

In parallel, we prepared complex three-dimensional monoand dual-species biofilms for $24 \mathrm{~h}$ on a $3 \mathrm{D}$ cellulose matrix model recently optimized and described by our group (Townsend et al., 2016). After growth, biofilms were gently washed with PBS before being treated with $40 \mathrm{mg} / \mathrm{L}$ of miconazole for a further $24 \mathrm{~h}$. Following treatment, biofilms were washed before being sonicated for $5 \mathrm{~min}$ in $1 \mathrm{~mL}$ of $\mathrm{PBS}$ at $35 \mathrm{kHz}$ in an ultrasonic water-bath (Fisher Scientific, Leicestershire, UK) to remove biofilm cells, and the viability of the biofilms after treatment was determined using species-specific live/dead PCR, as described above.

\section{Galleria mellonella Survival Assays}

The pathogenicity of C. albicans, S. aureus, and co-infection was assessed using the Galleria mellonella killing assay as previously described (Rajendran et al., 2015). Sixth instar G. mellonella larvae (Livefoods Direct Ltd, UK) with a body weight between 200 and $300 \mathrm{mg}$ were used. For each group, 30 larvae were injected directly into the hemocoel at a concentration of $5 \times 10^{5} \mathrm{CFU} /$ larvae/organism $\left(1 \times 10^{6} \mathrm{CFU} /\right.$ larvae total for co-infection) through the hindmost right proleg region, using a $50 \mu \mathrm{L}$ Hamilton syringe with a $26 \mathrm{~g}$ needle. Larvae were then placed in Petri dishes at $37^{\circ} \mathrm{C}$, with survival being monitored across a $48 \mathrm{~h}$ period. Larvae were considered dead when they displayed no movement in response to touch and showed severe melanisation within the cuticle as previously described (Sherry et al., 2014). To determine fungal and bacterial burden after $24 \mathrm{~h}$ post-infection, three representative larvae from each group were homogenized and DNA extracted as described elsewhere (Rajendran et al., 2015). The fungal and bacterial burden, expressed as CFE (CFE/100 mg body weight), was then assessed using species-specific primers and standard curves as described above. For antimicrobial treatment assays, larvae were administered with miconazole in the hindmost left proleg region $2 \mathrm{~h}$ post-infection of either mono- or dualspecies co-infection. A miconazole concentration of $75 \mathrm{mg} / \mathrm{kg}$ body weight was used throughout this experiment. Appropriate uninfected, drug only and vehicle controls were included for each experiment.

\section{Quantification of eDNA Release}

The quantity of eDNA released into the biofilm supernatant and ECM from C. albicans, S. aureus, and dual-species biofilms was measured using a microplate fluorescence assay (MFA), as previously described (Rajendran et al., 2014). Standardized cells $\left(1 \times 10^{6}\right.$ cells $\left./ \mathrm{mL}\right)$ were seeded in a black 96 -well microtiter plate (Corning, NY, USA) and SYBR ${ }^{\circledR}$ Green I (Invitrogen, Paisley, UK) added to the cells at a ratio of 1:10. The binding of this dye results in fluorescence that is directly proportional to the level of eDNA. These levels were then measured after 6,12 , and $24 \mathrm{~h}$ of growth using a microtiter plate reader at excitation and emission wavelengths of 485 and $518 \mathrm{~nm}$, and eDNA quantified in comparison to a standard curve. Next, in order to determine the quantity of eDNA contributing to the ECM the biomass of $24 \mathrm{~h}$ grown biofilms was removed using a cell scraper and then treated with $0.2 \mathrm{M}$ EDTA to extract the ECM. Samples were then centrifuged at 10,000 $\times g$ and EDTA supernatants recovered. The quantity of ECM associated eDNA was then calculated using the MFA described above. In addition, DNA was precipitated from the isolated matrix using ammonium acetate precipitation and speciesspecific eDNA was quantified using qPCR as described above, using fungal specific $18 \mathrm{~S}$ and bacteria specific $16 \mathrm{~S}$ primers (Table 1). 
TABLE 1 | Primer sequences used for qPCR.

\begin{tabular}{|c|c|c|}
\hline Primer & Sequence $\left(5^{\prime}-3^{\prime}\right)$ & Reference \\
\hline Candida albicans & F - GAGCGTCGTICTCCCTCAAACCGCTGG R - GGTGGACGTTACCGCCGCAAGCAATGTT & Alves et al., 2014 \\
\hline Staphylococcus aureus & F - ATTTGGTCCCAGTGGTGTGGGTAT R - GCTGTGACAATTGCCGTTTGTCGT & O’Donnell et al., 2016 \\
\hline $18 S$ & F - CTCGTAGTTGAACCTTGGGC R - GGCCTGCTITGAACACTCTA & Sherry et al., 2016 \\
\hline $16 S$ & F - CGCTAGTAATCGTGGATCAGAATG R - TGTGACGGGCGGTGTGTA & Sherry et al., 2016 \\
\hline
\end{tabular}

\section{Staphylococcus aureus Mycofilm Adherence Assay}

For adhesion, $24 \mathrm{~h}$ grown $C$. albicans biofilms were treated \pm DNase I [130 and $650 \mathrm{mg} / \mathrm{L}$ (Sigma-Aldrich, Dorset, $\mathrm{UK})$ ] prepared in a buffer solution containing $0.15 \mathrm{M} \mathrm{NaCl}$ with $5 \mathrm{mM} \mathrm{MgCl}_{2}$ for $4 \mathrm{~h}$ at $37^{\circ} \mathrm{C}$. S. aureus cells were standardized to $1 \times 10^{6} \mathrm{CFU} / \mathrm{mL}$ before being stained with $20 \mu \mathrm{M}$ SYTO9 ${ }^{\circledR}$ for $20 \mathrm{~min}$, prior to excess dye being removed by centrifugation. Biofilms were then washed again before the addition of the fluorescently stained $S$. aureus cells to the C. albicans biofilm for $90 \mathrm{~min}$. After incubation, biofilms were washed again to remove any non-adherent $S$. aureus cells and SYTO9 ${ }^{\circledR}$ fluorescence quantified using plate reader at an excitation and emission wavelengths of 485 and $518 \mathrm{~nm}$, respectively. The number of adherent cells was then quantified in comparison to a serially diluted $S$. aureus standard curve.

\section{Statistical Analysis}

Graph production, data distribution, and statistical analysis were performed using GraphPad Prism (version 5; La Jolla, CA, USA). Student $t$-tests were used to analyze experiments comparing independent sample data. Kaplan Meier survival curves were analyzed using the log-rank test. Statistical significance was achieved if $P<0.05$.

\section{RESULTS}

\section{Staphylococcus aureus Utilizes Candida albicans Mycofilms as a Structural Scaffold}

First, we wanted to determine the basis of how C. albicans and $S$. aureus interact within a biofilm environment. We purposely used the Newman's strain of $S$. aureus that has a defective biofilm phenotype (Beenken et al., 2003; Supplementary Figure 1), working with the hypothesis that C. albicans biofilm structure positively influenced its ability to colonize and form biofilms irrespective of host derived substrates. Mono- and dual-species biofilms were quantified at early (90 min), intermediate $(6 \mathrm{~h})$, and mature $(24 \mathrm{~h})$ stages of biofilm growth using the $\mathrm{CV}$ biomass assay (Figure 1A). It was shown that dual-species biofilms contained significantly more biomass than either of the mono-species organism's biofilms at $90 \mathrm{~min}, 6$ and $24 \mathrm{~h}$ $(p<0.001)$. A sensitive quantitative molecular approach was then used in order to assess both viability and composition of each species within the dual-species biofilm throughout maturation. After $90 \mathrm{~min}$ no statistical differences were observed in the overall total and viable number of $C$. albicans between the mono- and dual-species biofilms $(p=0.1021)$, though a 2.76and 3.92-fold increase in the total and live number of $S$. aureus cells was observed within the dual-species biofilm, respectively (Figure 1B). When scrutinized visually we observe C. albicans cells existing as germ tubes with single and small clusters of S. aureus cells adhered to these structures (Figure 1C). As the biofilm progressed to the intermediate phase of growth at $6 \mathrm{~h}$ no difference was observed in the total and live number of C. albicans cells between mono- and dual-species biofilm cultures (Figure 1B). Though, a significant $S$. aureus increase within the dual-species biofilm was observed ( $p<0.01,24.11$-fold), which is reinforced microscopically where we observe enhanced colonies of clustered $S$. aureus cells attached to the hyphae of C. albicans (Figure 1C). Next, when we evaluated fully matured $24 \mathrm{~h}$ biofilms we observe similar trends as above (Figure 1B), with no statistical difference between the total numbers of C. albicans cells between both biofilms. Intriguingly though, when we quantified the number of dead cells (total cells minus live cells) we observed a statistically significant increase in the quantity of dead C. albicans cells in the dual-species biofilm $(p<0.01$, 2.52 -fold). Conversely, $S$. aureus was significantly increased in the dual-species biofilm compared to mono-species biofilms, with total and viable cells number increasing 5.85- and 3.66-fold, respectively $(p<0.05)$. Visually, these dual-species biofilms were characterized by extensively spread micro-colonies of $S$. aureus biofilm cells attached to and interspersed throughout the dense network of C. albicans hyphal growth (Figure 1C). Collectively, these data suggest that these two microorganisms display a level of synergy given their intimate physical relationship, where candidal mycofilms serve as the base substrate for $S$. aureus colonization.

\section{Dual-Species Biofilms Decrease Miconazole Efficacy In vitro}

First, we investigated the sensitivity of simple mono- and dualspecies planktonic and sessile cells using standardized metabolic based methodologies. It was shown that the PMIC for $S$. aureus was $1 \mathrm{mg} / \mathrm{L}$ and $C$. albicans and dual-species were $4 \mathrm{mg} / \mathrm{L}$. The $\mathrm{SMIC}_{80}$, i.e., the concentration that inhibited $80 \%$ metabolic activity of the biofilm, was 10 -fold greater for each biofilm tested, with $S$. aureus mono-species biofilms at $10 \mathrm{mg} / \mathrm{L}$, whereas for both the $C$. albicans mono-species and the dual-species biofilms at $40 \mathrm{mg} / \mathrm{L}$ (Table 2).

Next, we evaluated mono- and dual- species biofilms within a more complex 3D wound model determined their sensitivity against a $24 \mathrm{~h}$ treatment of miconazole $\left(\mathrm{SMIC}_{80}=40 \mathrm{mg} / \mathrm{L}\right)$. No significant differences were observed for $C$. albicans 
A

90 minutes

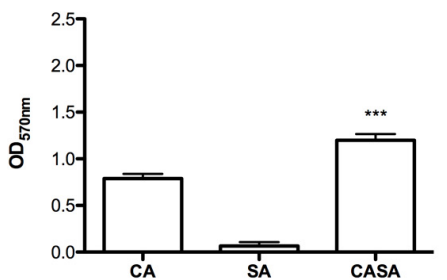

B
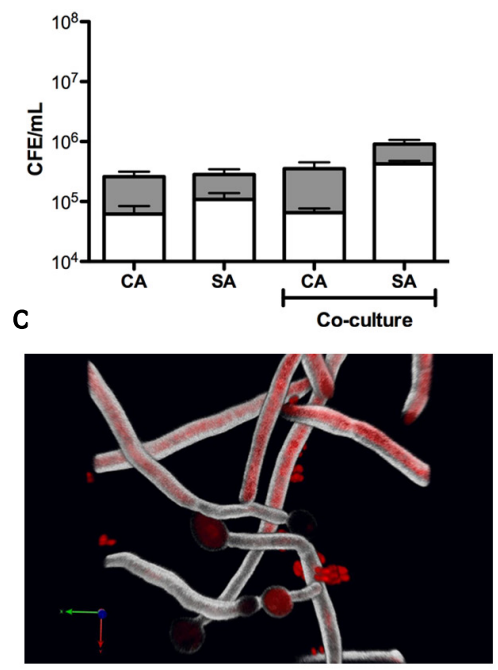

6 hours
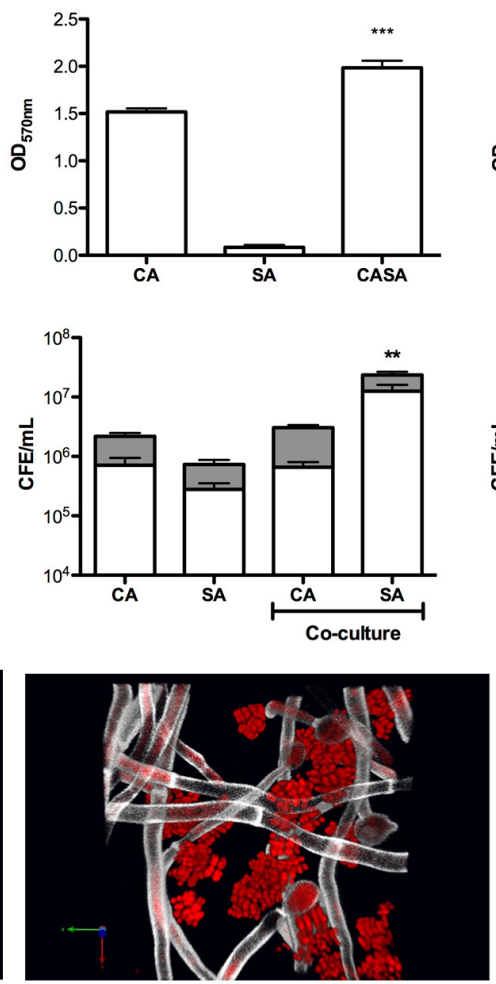

24 hours
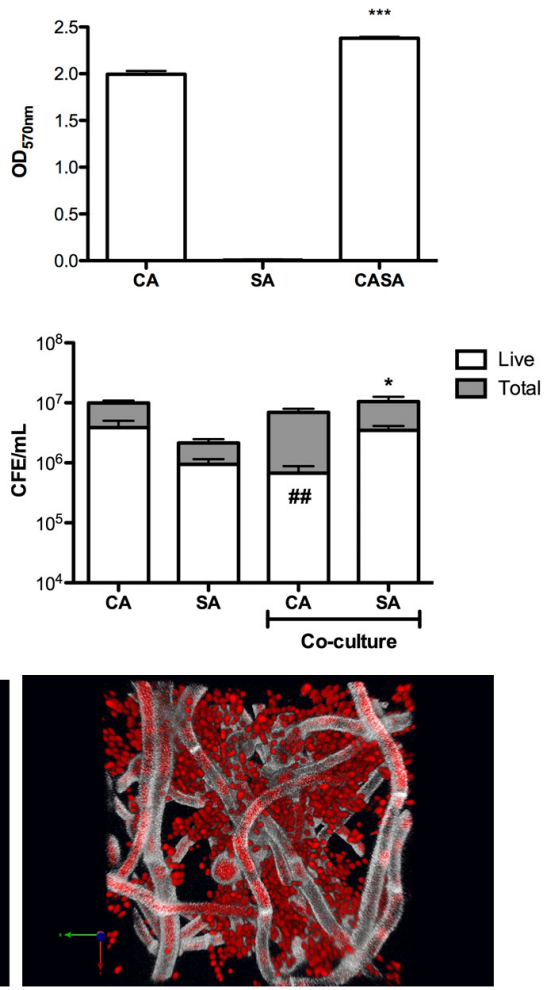

FIGURE 1 | Candida albicans mycofilms facilitates Staphylococcus aureus biofilm formation. Mono- and dual-species biofilms were standardized $1 \times 10^{6}$ CFU/mL and grown on 96-well plates for $90 \mathrm{~min}, 6$ and $24 \mathrm{~h}$. Biofilms were then washed with phosphate buffered saline (PBS) and biomass assessed using the crystal violet assay (A). Standardized biofilms were grown before being sonicated to remove the biomass. Live/dead PCR was then used to extract DNA and determine total and live colony forming equivalents (CFE) from mono- and dual-species biofilms (B). Biofilm morphology was then analyzed using CSLM. Biofilms were grown before being fluorescently stained using calcofluor white and SYTO9® ${ }^{\circledR}$ dyes. Resulting biofilms were then viewed on a Leica SP5 laser scanning confocal microscope and images were then processed and analyzed using Volocity 3D Image Analysis Software (C). Results represent data from three independent occasions. Statistical analysis compares dual-species biofilms to their mono-species equivalent $\left({ }^{*} p<0.05,{ }^{* *} p<0.01,{ }^{* * *} p<0.001\right)$. ${ }^{\# \#} p<0.01$, compares dead cells between dual- and mono-species biofilms.

TABLE 2 | Sessile MIC for miconazole in the presence and absence of hydrolytic enzymes.

\begin{tabular}{|c|c|c|c|c|}
\hline \multicolumn{5}{|c|}{ Minimum inhibitory concentration (mg/L) } \\
\hline & \multicolumn{4}{|c|}{$\mathrm{SMIC}_{80}$} \\
\hline & Miconazole & Miconazole + DNase & Miconazole + Lyticase & Miconazole + Chitinase \\
\hline C. albicans (CA) & 40 & 40 & 40 & 40 \\
\hline S. aureus (SA) & 10 & 10 & 10 & 10 \\
\hline $\mathrm{CA}+\mathrm{SA}$ & 40 & 20 & 40 & 40 \\
\hline
\end{tabular}

post-treatment between mono- and dual-species biofilms (Figure 2). However, there was decreased sensitivity observed in $S$. aureus when grown in the dual-species biofilm, with a significant 3.52 -fold increase in the quantity of live cells post-treatment ( $p<0.01$; Figure 2$)$.

\section{Dual-Species Biofilms Decrease Miconazole Efficacy In vivo}

In order to first establish whether any co-operative pathogenicity exists between both organisms in vivo, a G. mellonella infection model was utilized. Inoculation of larvae with C. albicans was shown to kill approximately 60 and $80 \%$ of larvae after 24 and $48 \mathrm{~h}$ post-infection, respectively. Conversely, larvae coinfected with both $C$. albicans and $S$. aureus demonstrated an 80 and $100 \%$ mortality rate after 24 and 48 h, respectively. This was a significant increase compared to both $C$. albicans $(p<0.05)$ only and $S$. aureus only $(p<0.001)$, the latter of which was avirulent at the chosen cell concentration (Figure $3 \mathbf{A}$ and Supplementary Figure 2). Three selected larvae were then chosen to determine the microbial burden at $24 \mathrm{~h}$ post-infection. 


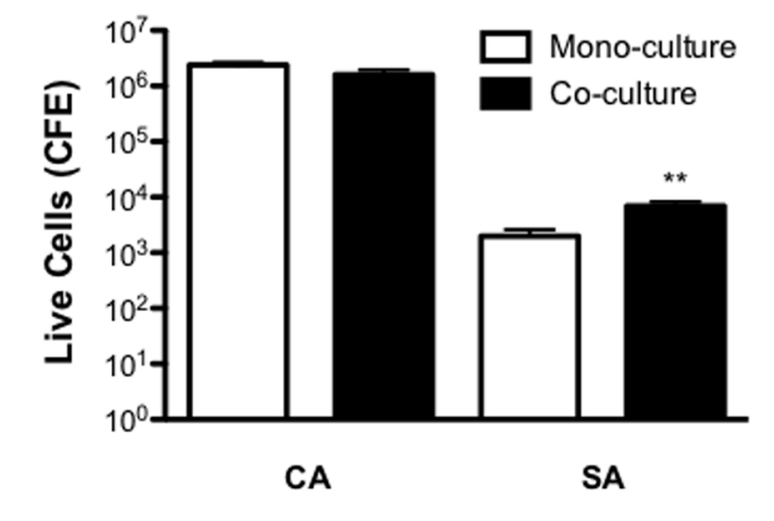

FIGURE 2 | Inter-kingdom interactions decrease Staphylococcus aureus sensitivity to miconazole. Biofilms were grown for $24 \mathrm{~h}$ on a cellulose matrix based hydrogel model before being washed with PBS and treated with $40 \mathrm{mg} / \mathrm{L}$ of miconazole for a further $24 \mathrm{~h}$. After treatment, the cellulose matrix was removed and sonicated to dislodge the biofilm biomass. Live/dead PCR was then used to extract DNA and quantify total and live CFE. Data is presented as the CFE of live cells comparing treated mono- and dual-species biofilms. Data represents duplicate samples from three independent time points with significance achieved with ${ }^{* *} p<0.01$.

Results showed that $C$. albicans CFE counts remained at equivalent levels in mono- and dual-infection. Whereas, as observed with the in vitro data the quantity of $S$. aureus was significantly enhanced 11.1-fold when co-infected with C. albicans $(p<0.05$; Figure 3B). These data indicate that when in combination the pathogenic outcome is synergised in coinfection, and given that $S$. aureus is quantitatively enhanced then this would suggest $C$. albicans supports and enhances its growth.

Next, we tested the efficacy of miconazole against this synergised co-infection using the G. mellonella infection model. Upon $2 \mathrm{~h}$ post-infection larvae were administered with miconazole and mortality monitored over a $48 \mathrm{~h}$ period. Results showed that after $24 \mathrm{~h}$, over $75 \%$ of C. albicans infected larvae remained alive after miconazole treatment, and almost 50\% survived $48 \mathrm{~h}$ after infection. All larvae remained alive at $48 \mathrm{~h}$ post-inoculation with $S$. aureus. However, co-infection with both organisms resulted in a loss of susceptibility to miconazole, with mortality reaching almost $70 \%$ after $24 \mathrm{~h}$ and $>85 \%$ at 48 h postinfection, a significant difference in larvae survival compared to C. albicans $(p<0.01)$ and S. aureus $(p<0.001)$ alone (Figure 3C).

\section{Extracellular DNA Promotes Stability to the ECM of Dual-Species Biofilms}

When analyzed visually through SEM, clusters of $S$. aureus biofilm can be seen embedded within C. albicans mycofilms and associated with ECM (Figure 4). Given that ECM is an important characteristic associated with antifungal resistance, we hypothesized that degradation of ECM components in combination with miconazole would enhance biofilm sensitivity. It was shown that degradation of eDNA, $\beta$-1,3-glucan and chitin had no effect on the $\mathrm{MIC}_{80}$ value for C. albicans and $S$. aureus mono-species biofilms. However, when these same components were degraded by DNase in the dual-species biofilm, we observed a twofold increase in miconazole sensitivity, but no change when treated in combination with the other enzymes (Table 2).

To determine whether C. albicans positively influenced this dual-species interaction we next investigated the mechanistic contribution of the ECM, hypothesizing that eDNA may be pivotal factor in supporting the dual-species interactions, as has been shown in mono-species C. albicans biofilms (Rajendran et al., 2014). Figure 5A shows an increase of eDNA release into the biofilm supernatant from dual-species biofilm compared to a C. albicans biofilm in a time dependent manner. Throughout the assessed time points, mono-species $S$. aureus biofilms eDNA levels were below detectable limit. After $90 \mathrm{~min}$ of biofilm development eDNA levels of all tested groups remained below a detectable level. After $6 \mathrm{~h}$ of growth, there was no significant difference between $C$. albicans and dual-species eDNA release $(p=0.4296)$. At $12 \mathrm{~h}$, a significant increase in the quantity of eDNA released from the dual-species biofilm was observed (2.28fold, $p<0.05$ ). After $24 \mathrm{~h}$ the quantity of eDNA released from C. albicans biofilms plateaued, whereas the dual-species biofilm continued to rise, with a significant increase ( $p<0.01,4.40$-fold).

Finally, we next assessed if this secreted eDNA contributed to the biofilm structure by measuring the eDNA quantity within the ECM. It was shown that the ECM associated eDNA significantly increased 2.15-fold when comparing the dual-species biofilm to the $C$. albicans biofilm $(P<0.01$; Figure 5B). We then used qPCR to analyze the species contribution to this increase, showing that in the dual-species biofilm both organisms released significantly more eDNA than their mono-species counterparts. The quantity of $C$. albicans eDNA significantly increased from 408.5 to $611.1 \mathrm{pg} / \mathrm{mL}$ in the co-culture $(p<0.05)$, with the $S$. aureus eDNA also significantly increasing 4.7-fold $(p<0.001$; Figure 5C). Due to the increase of eDNA in the dual-species biofilm we then investigated its role in supporting the adhesion of $S$. aureus to a mature C. albicans biofilm. It was shown that a $4 \mathrm{~h}$ DNase treatment at $130 \mu \mathrm{g} / \mathrm{mL}$ did not have any detectable effect on $S$. aureus adhesion compared to the control, whereas when the DNase concentration was increased $(650 \mu \mathrm{g} / \mathrm{mL})$ we observed a significant $26.5 \%$ reduction in the number of $S$. aureus cells that adhered to the $C$. albicans biofilm $(P<0.05$; Figure 5D).

\section{DISCUSSION}

Polymicrobial infections are increasingly recognized as a clinically important entities due to altered patient prognosis that results in increased hospital stays and decreased antimicrobial efficacy (Sancho et al., 2012). In this manuscript, we aimed to characterize the inter-kingdom interactions between the two most common nosocomial pathogens C. albicans and S. aureus, and subsequently evaluate miconazole in vitro and in vivo sensitivities, an antifungal with reported cross-kingdom efficacy. Using this strategy it was shown that $S$. aureus is able to use C. albicans as a physical scaffold to colonize and form biofilms upon an existing biofilm, a phenomenon we have termed as mycofilms. We also report a novel role for eDNA in promoting 

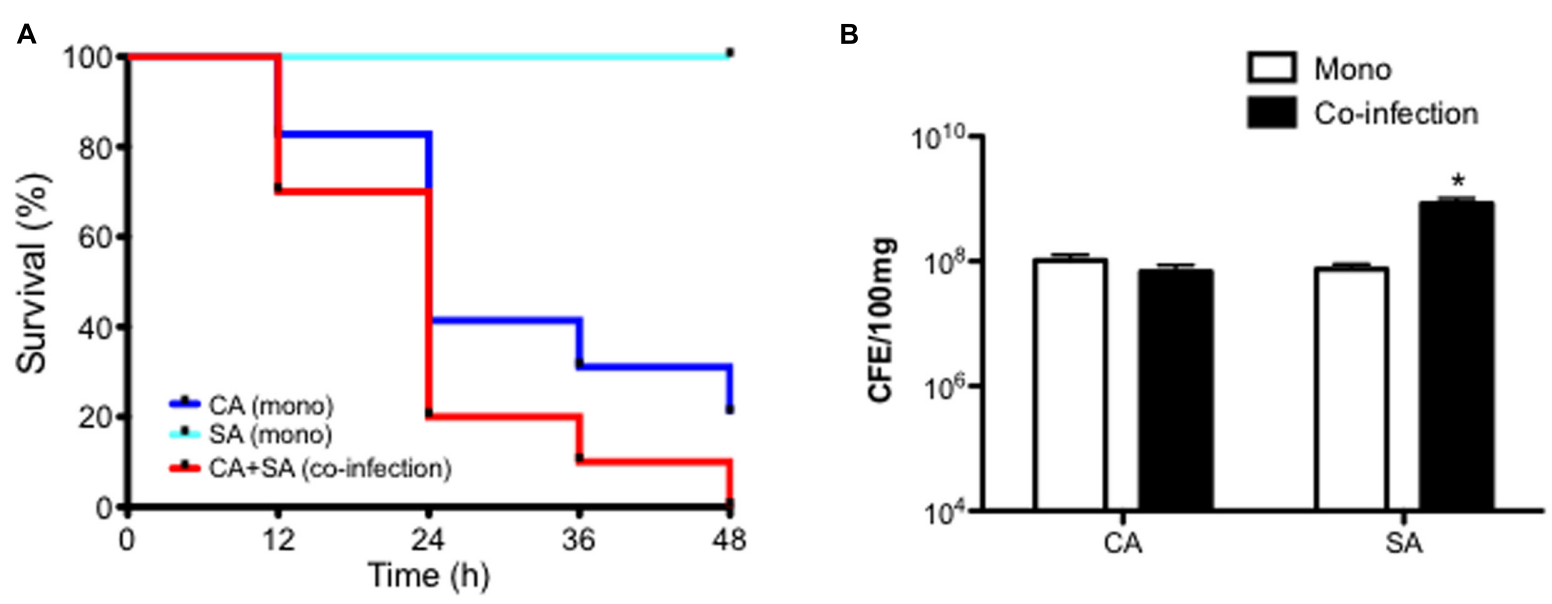

C

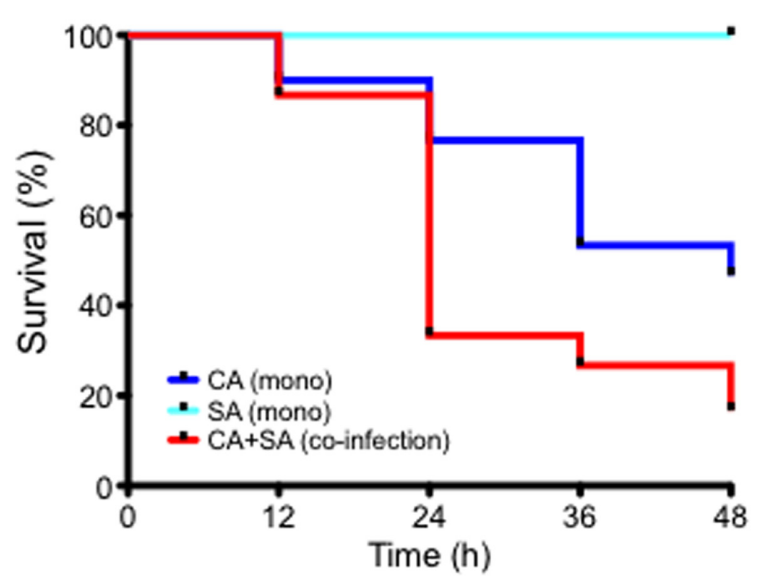

FIGURE 3 | Candida albicans and Staphylococcus aureus display synergistic pathogenicity and reduced miconazole sensitivity in vivo. C. albicans $(\mathrm{CA})$ and S. aureus (SA) were standardized to $5 \times 10^{5} \mathrm{CFU} /$ larvae and administered to larvae as a mono or co-infection (CA+SA) with percentage survival monitored across a $48 \mathrm{~h}$ period and data presented using a Kaplan Meier plot. Data are derived from three independent groups of 10 larvae with significance $\left(^{*} p<0.05\right.$, ${ }^{* *} p<0.001$ ) determined using the log-rank test in comparison between C. albicans and S. aureus alone and co-infection (A). After 24 h post-infection, representative larvae were snap frozen in liquid nitrogen and DNA extracted. Microbial burden was then determined using qPCR and presented as CFE (B). Data are derived from three larvae with ${ }^{*} p<0.05$. Upon 2 h post-infection of larvae with $5 \times 10^{5} \mathrm{CFU} /$ larvae with $\mathrm{CA}$, SA, and co-infection (CA+SA), larvae were administered with $75 \mathrm{mg} / \mathrm{kg}$ of miconazole. Percentage survival was monitored across a $48 \mathrm{~h}$ period and represented with a Kaplan Meier plot (C). Data represents results from three independent groups of 10 larvae with significance achieved $\left(* * p<0.01,{ }^{* * *} p<0.001\right)$ comparing C. albicans, S. aureus, and co-infection using the log-rank test.

the stability of C. albicans/S. aureus biofilms that augments biofilm mediated resistance to miconazole.

An initial objective from this series of studies was to create a reliable functional assay to quantify and characterize the interaction between $C$. albicans and $S$. aureus. It was our hypothesis based on previous studies that $C$. albicans hyphae are integral to supporting $S$. aureus biofilm formation (Peters et al., 2012b). To test this hypothesis we used the biofilm defective $S$. aureus Newman strain to determine whether the presence of $C$. albicans could positively enhance its ability to colonize and form biofilms. Indeed, our hypothesis was confirmed with the observation that single $S$. aureus cells and small clusters adhere to $C$. albicans germ tubes after only 90 min of growth, while synchronously enhancing overall biomass, significantly increasing throughout biofilm maturation. This is in line with previous reports predicting the initiation of polymicrobial biofilm occurs upon initial C. albicans germ tube formation (Harriott and Noverr, 2009), and continues as the biofilm becomes fully mature (Peters et al., 2010). Given that the ALS3 gene is highly expressed during early phases of $C$. albicans filamentous growth during biofilm formation (Sherry et al., 2014), then it is unsurprising that this key biofilm adhesin is involved in mediating the initial attachment of bacteria within the polymicrobial biofilm (Peters et al., 2012b). Als3p shares between 80 and $\geq 95 \%$ homology with collagen binding and clumping factor proteins of $S$. aureus (Sheppard et al., 2004), therefore the cellular basis for these interactions may be reciprocal. Indeed, the anti-candidal vaccine 


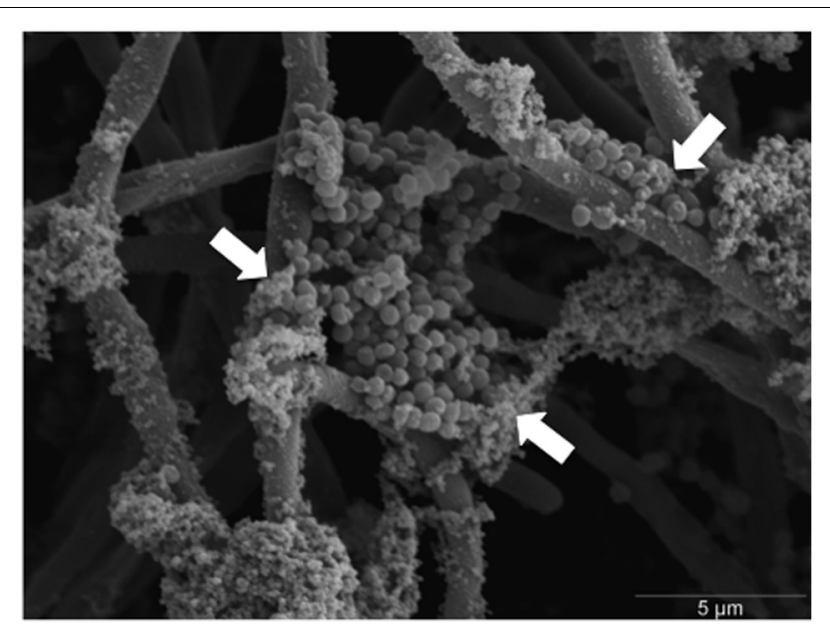

FIGURE 4 | Scanning electron micrograph of S. aureus colonizing C. albicans hyphae within dual-species biofilms. Dual-species biofilms were grown for $24 \mathrm{~h}$ before being fixed, processed, and imaged using a JEOL-JSM 6400 scanning electron microscope. S. aureus colonies can be seen adhering and embedded within the hyphal meshwork of $C$. albicans. White arrows indicate clusters of $S$. aureus colonies encased within extracellular matrix $(E C M)$. Scale bar represents $5 \mu \mathrm{m}$ at $\times 5000$ magnification.

derived from the Als3p also protects against $S$. aureus infection in a murine model (Spellberg et al., 2008). Interestingly, the use of the biofilm defective Newman's strain of $S$. aureus suggests that this species does not necessarily require the full armamentarium of biofilm-associated genes, as the presence of a scaffold of C. albicans hyphae is capable of facilitating the process, which may explain why these organisms as so frequently co-isolated (Beenken et al., 2003). This may also explain why there is such heterogeneity in terms of $S$. aureus biofilm formation (Smith et al., 2008), a phenomenon also evident in C. albicans biofilm formation (Sherry et al., 2014; Rajendran et al., 2016a).

As C. albicans biofilm maturation progressed the quantities of $S$. aureus was significantly enhanced, though notably there was a significant increase in the number of dead C. albicans cells, suggesting that $S$. aureus is able to utilize $C$. albicans in some undefined way through this close interaction. Metabolomic analysis of the interaction enabled us to recently identify that $S$. aureus is able to consume sterols, products also found in the secretome of C. albicans biofilms (Weidj et al., 2016). Given that sterols have been shown to promote $S$. aureus growth (Shine et al., 1993), then this suggests that sterol consumption is a contributing factor to its increased growth rates in the dual-species biofilm. Other metabolites, such as the C. albicans secreted QS compound farnesol has also been shown to modulate $S$. aureus behavior through inhibiting lipase activity and biofilm formation, as well as improving its sensitivity to gentamicin (Jabra-Rizk et al., 2006; Kuroda et al., 2007; Unnanuntana et al., 2009). Collectively, these data indicate that these organisms have the capacity to interact with one another at the molecular level. Indeed, our studies demonstrated that in the G. mellonella model there is enhanced virulence in the dual-species infection compared to both mono-species infections, which is in agreement with previous in vivo murine studies also describing enhanced virulence of $C$. albicans and S. aureus co-infection (Carlson, 1983; Peters and Noverr, 2013). One interesting hypothesis is that $S$. aureus utilize C. albicans hyphae as a transportation mechanism, much like a needlestick injection, to mediate systemic infection throughout the host (Schlecht et al., 2015). This may be the reasoning behind previous findings reporting that bacteria were recovered from the kidneys of an oral candidiasis co-infection model, whereas in the monoinfection model no. S. aureus, were detected (Kong et al., 2015).

Given the clinical implications of the co-infection, we were then interested in evaluating miconazole as a treatment possibility, which has been shown to possess cross-kingdom efficacy (Boyen et al., 2012). Miconazole functions via a dual mechanism by primarily interfering with lipid membranes through the inhibition of ergosterol synthesis resulting in an accumulation of sterol by-products that consequently prevents cell growth (Vanden Bossche et al., 1990). Additionally, it functions in the stimulation of reactive oxygen species (ROS) promoting cell death (Kobayashi et al., 2002; Vandenbosch et al., 2010). It was shown that 10 -fold greater concentrations of miconazole were required to induce an effect against the monoand dual-species biofilms compared to planktonic combinations, and the MIC levels in dual-species were ultimately defined by $C$. albicans $(40 \mathrm{mg} / \mathrm{L})$. As this was a simplified model on plastics we also utilized a more representative $3 \mathrm{D}$ model to determine the viable composition (Townsend et al., 2016), where we showed significantly more viable $S$. aureus than the mono-culture model. This resistance was also translated into the in vivo model where reduced sensitivity was clearly demonstrated, which we propose is facilitated by the candidalmediated protection, and in particular the ECM as has been reported elsewhere (De Brucker et al., 2015; Kong et al., 2016).

The presence of $C$. albicans ECM within the mixedspecies biofilm has been shown to protect $S$. aureus from vancomycin treatment through concentrations escalating as high as $1600 \mathrm{mg} / \mathrm{mL}$ (Harriott and Noverr, 2009). A recent study has elaborated on this work, identifying fungal $\beta$-1,3-glucan as a matrix component to which $S$. aureus can encase itself within and decrease its susceptibility to vancomycin (Kong et al., 2016). The C. albicans ECM ( $\beta$-1,3-glucans) has also been shown to increase resistance to ofloxacin within $E$. coli dual-species biofilms (De Brucker et al., 2015). Interestingly though, of all C. albicans ECM constituents tested, degradation of eDNA using DNase was shown to be the second most effective way of reducing ofloxacin tolerance in this dualspecies model. The presence of biofilm associated eDNA has been identified and characterized in a range of bacterial and fungal pathogens including both $S$. aureus and C. albicans (Lister and Horswill, 2014; Rajendran et al., 2014). S. aureus eDNA release has been extensively characterized (Memmi et al., 2012), though eDNA release in the mixed species biofilm is less well defined. Studies between C. albicans and Pseudomonas 
A

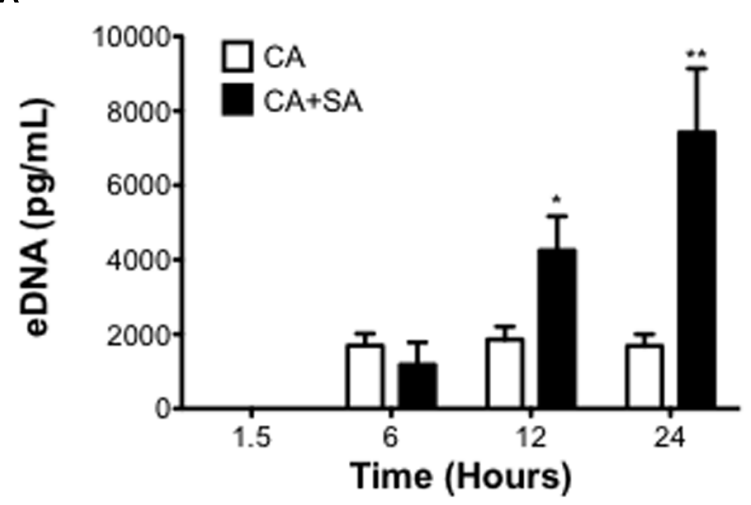

C

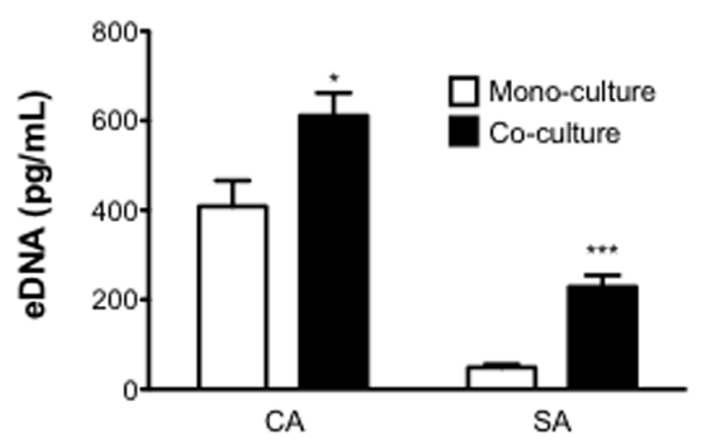

B

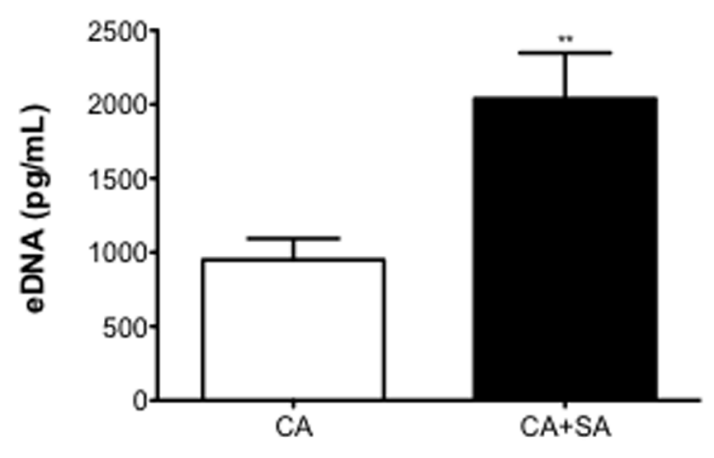

D

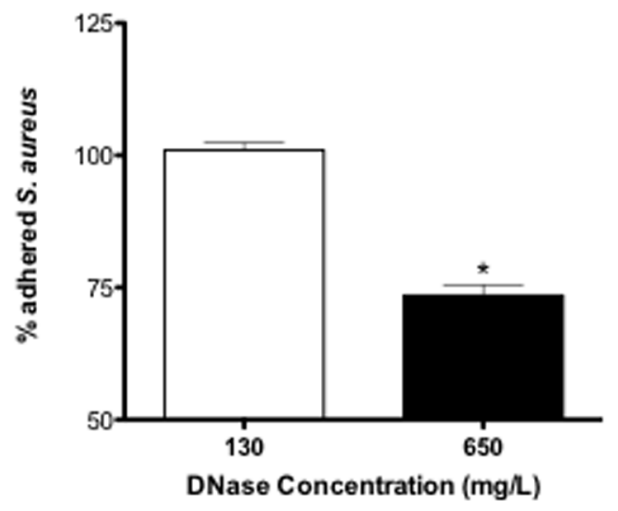

FIGURE 5 | Extracellular DNA contributes to inter-kingdom pathogenicity. Mono- and dual-species biofilms were seeded at $1 \times 10^{6} \mathrm{CFU} / \mathrm{mL}$ in black $96-$ well plates and eDNA release at 1.5, 6, 12, and $24 \mathrm{~h}$ measured using a SYBR ${ }^{\circledR}$ Green 1 based microplate fluorescence assay (MFA) in comparison to a standard curve (A). Biofilms were washed with $0.2 \mathrm{M}$ EDTA to remove the ECM and resulting eDNA quantified using the MFA described above in comparison to a standard curve (B). ECM associated DNA was then precipitated from matrix extracts and species contributions were analyzed using qPCR (C). C. albicans only biofilms were grown for $24 \mathrm{~h}$ in black 96 -well plates. After washing biofilms were then treated with either 130 or $650 \mathrm{mg} / \mathrm{L}$ of DNase for $4 \mathrm{~h}$. SYTOg ${ }^{\circledR}$ stained S. aureus cells $\left(1 \times 10^{6}\right.$ $\mathrm{CFU} / \mathrm{mL}$ ) were then added to the biofilm and incubated for 90 mins before being fluorescently quantified in comparison to an vehicle control treated biofilm (D). Data represents duplicate samples from three independent experiments $\left({ }^{*} p<0.05,{ }^{* *} p<0.01,{ }^{* * *} p<0.001\right)$.

aeruginosa revealed that secreted bacterial components are able to induce fungal cell lysis, as well as modulating fungal virulence (Gibson et al., 2009). While it is unlikely that S. aureus is able to secrete reciprocal components to $P$. aeruginosa, it is conceivable that as nutrients become sparse within mature biofilms $S$. aureus may scavenge off the fungal cell wall to utilize its own growth, as was reported through our metabolomic analyses (Weidj et al., 2016). When we examined the release of eDNA into the biofilm supernatant it was shown to be phase dependent, with enhanced eDNA release from the dual-species biofilms after $12 \mathrm{~h}$, continuing to increase as biofilms matured, and this increased for both organisms within the biofilm. In fact, exogenous $S$. aureus genomic DNA has been shown to positively impact C. albicans biofilm formation (Sapaar et al., 2014).

This study demonstrates that enzymatic degradation of eDNA of a $C$. albicans biofilm negatively affects $S$. aureus adhesion, highlighting therapeutic potential. The effective disruption of $C$. albicans matrix components to enhance antimicrobial efficacy has also been demonstrated within in vitro mono- and dual-species biofilms (Nett et al., 2007; Rajendran et al., 2014; De Brucker et al., 2015; Kong et al., 2016). The ability to de-stabilize biofilms through degradation of eDNA make it an attractive therapeutic target, especially in polymicrobial biofilms where they can be used to sensitize co-colonizing bacteria. Further studies of these agents are required to validate their efficacy in vivo. The DNase Pulmozyme ${ }^{\circledR}$ is currently used in combination with antibiotics for cystic fibrosis treatment, with significant reduction in the prevalence of $S$. aureus found within treated patients compared to untreated controls (Frederiksen et al., 2006).

To summarize, this manuscript reports the close association of two commonly co-isolated nosocomial pathogens, and indicates that $S$. aureus can utilize $C$. albicans biofilm architecture to its advantage. We also demonstrate that miconazole resistance within dual-species biofilms is supported by eDNA. Further exploration of this intriguing relationship between these two organisms will aid to a better understanding of their frequent coisolation from the human host, and may provide novel avenues 
of possible therapeutic strategies to combat inter-kingdom biofilm infections, particularly those infections of a topical nature such as wounds, diabetic foot ulcers, and angular cheilitis.

\section{AUTHOR CONTRIBUTIONS}

RK, RR, JH, ET, and BS participated in study design and experimental procedures and were responsible for preparation of the manuscript. $\mathrm{KB}$ and SL contributed to study design and manuscript preparation. OM participated in study design and confocal microscopy imaging and analysis and manuscript preparation. WM and CW participated in study design, analysis of the data, and contributed to the manuscript. GR conceived the study, participated in study design and was responsible for producing the final manuscript. All authors have read and approved the final manuscript.

\section{REFERENCES}

Alves, C. T., Silva, S., Pereira, L., Williams, D. W., Azeredo, J., and Henriques, M. (2014). Effect of progesterone on Candida albicans vaginal pathogenicity. Int. J. Med. Microbiol. 304, 1011-1017. doi: 10.1016/j.ijmm.2014.07.004

Beenken, K. E., Blevins, J. S., and Smeltzer, M. S. (2003). Mutation of sarA in Staphylococcus aureus limits biofilm formation. Infect. Immun. 71, 4206-4211. doi: 10.1128/IAI.71.7.4206-4211.2003

Boyen, F., Verstappen, K. M., De Bock, M., Duim, B., Weese, J. S., Schwarz, S., et al. (2012). In vitro antimicrobial activity of miconazole and polymyxin B against canine meticillin-resistant Staphylococcus aureus and meticillinresistant Staphylococcus pseudintermedius isolates. Vet. Dermatol. 23, e70. doi: 10.1111/j.1365-3164.2012.01040.x

Carlson, E. (1983). Effect of strain of Staphylococcus aureus on synergism with Candida albicans resulting in mouse mortality and morbidity. Infect. Immun. 42, 285-292.

CLSI (2012). Reference Method for Broth Dilution Antifungal Susceptibility Testing Of Yeasts, Approved Standard - CLSI Document M27-A3, 3rd Edn. Wayne, PA: Clinical \& Laboratory Standards Institute.

De Brucker, K., Tan, Y., Vints, K., De Cremer, K., Braem, A., Verstraeten, N., et al. (2015). Fungal beta-1,3-glucan increases ofloxacin tolerance of Escherichia coli in a polymicrobial E. coli/Candida albicans biofilm. Antimicrob. Agents Chemother. 59, 3052-3058. doi: 10.1128/AAC.04650-14

Duthie, E. S., and Lorenz, L. L. (1952). Staphylococcal coagulase; mode of action and antigenicity. J. Gen. Microbiol. 6, 95-107. doi: 10.1099/00221287-6-1-2-95

Erlandsen, S. L., Kristich, C. J., Dunny, G. M., and Wells, C. L. (2004). Highresolution visualization of the microbial glycocalyx with low-voltage scanning electron microscopy: dependence on cationic dyes. J. Histochem. Cytochem. 52, 1427-1435. doi: 10.1369/jhc.4A6428.2004

Fonzi, W. A., and Irwin, M. Y. (1993). Isogenic strain construction and gene mapping in Candida albicans. Genetics 134, 717-728.

Frederiksen, B., Pressler, T., Hansen, A., Koch, C., and Hoiby, N. (2006). Effect of aerosolized rhDNase (Pulmozyme) on pulmonary colonization in patients with cystic fibrosis. Acta Paediatr. 95, 1070-1074. doi: 10.1080/08035250600752466

Gibson, J., Sood, A., and Hogan, D. A. (2009). Pseudomonas aeruginosa-Candida albicans interactions: localization and fungal toxicity of a phenazine derivative. Appl. Environ. Microbiol. 75, 504-513. doi: 10.1128/AEM.01037-08

Harriott, M. M., and Noverr, M. C. (2009). Candida albicans and Staphylococcus aureus form polymicrobial biofilms: effects on antimicrobial resistance. Antimicrob. Agents Chemother. 53, 3914-3922. doi: 10.1128/AAC.00657-09

Jabra-Rizk, M. A., Meiller, T. F., James, C. E., and Shirtliff, M. E. (2006). Effect of farnesol on Staphylococcus aureus biofilm formation and antimicrobial susceptibility. Antimicrob. Agents Chemother. 50, 1463-1469. doi: 10.1128/ AAC.50.4.1463-1469.2006

Kart, D., Tavernier, S., Van Acker, H., Nelis, H. J., and Coenye, T. (2014). Activity of disinfectants against multispecies biofilms formed by Staphylococcus aureus,

\section{FUNDING}

RR is supported by the Wellcome Trust Strategic Award for Medical Mycology and Fungal Immunology 097377/Z/11/Z.

\section{ACKNOWLEDGMENT}

We thank Margaret Mullin from the University of Glasgow for her expert assistance with scanning electron microscopy.

\section{SUPPLEMENTARY MATERIAL}

The Supplementary Material for this article can be found online at: http://journal.frontiersin.org/article/10.3389/fmicb. 2017.00258/full\#supplementary-material

Candida albicans and Pseudomonas aeruginosa. Biofouling 30, 377-383. doi: 10.1080/08927014.2013.878333

Klotz, S. A., Chasin, B. S., Powell, B., Gaur, N. K., and Lipke, P. N. (2007). Polymicrobial bloodstream infections involving Candida species: analysis of patients and review of the literature. Diagn. Microbiol. Infect. Dis. 59, 401-406. doi: 10.1016/j.diagmicrobio.2007.07.001

Kobayashi, D., Kondo, K., Uehara, N., Otokozawa, S., Tsuji, N., Yagihashi, A., et al. (2002). Endogenous reactive oxygen species is an important mediator of miconazole antifungal effect. Antimicrob. Agents Chemother. 46, 3113-3117. doi: 10.1128/AAC.46.10.3113-3117.2002

Kong, E. F., Kucharikova, S., Van Dijck, P., Peters, B. M., Shirtliff, M. E., and Jabra-Rizk, M. A. (2015). Clinical Implications of Oral Candidiasis: host tissue damage and disseminated bacterial disease. Infect. Immun. 83, 604-613. doi: 10.1128/IAI.02843-14

Kong, E. F., Tsui, C., Kucharikova, S., Andes, D., Van Dijck, P., and JabraRizk, M. A. (2016). Commensal Protection of Staphylococcus aureus against antimicrobials by Candida albicans biofilm matrix. MBio 7, e01365-16. doi: 10.1128/mBio.01365-16

Kuroda, M., Nagasaki, S., Ito, R., and Ohta, T. (2007). Sesquiterpene farnesol as a competitive inhibitor of lipase activity of Staphylococcus aureus. FEMS Microbiol. Lett. 273, 28-34. doi: 10.1111/j.1574-6968.2007.00772.x

Lin, Y. J., Alsad, L., Vogel, F., Koppar, S., Nevarez, L., Auguste, F., et al. (2013). Interactions between Candida albicans and Staphylococcus aureus within mixed species biofilms. BIOS 84, 30-39. doi: 10.1893/0005-3155-84.1.30

Lister, J. L., and Horswill, A. R. (2014). Staphylococcus aureus biofilms: recent developments in biofilm dispersal. Front. Cell Infect. Microbiol. 4:178. doi: 10. 3389/fcimb.2014.00178

Memmi, G., Nair, D. R., and Cheung, A. (2012). Role of ArlRS in autolysis in methicillin-sensitive and methicillin-resistant Staphylococcus aureus strains. J. Bacteriol. 194, 759-767. doi: 10.1128/JB.06261-11

Nett, J., Lincoln, L., Marchillo, K., Massey, R., Holoyda, K., Hoff, B., et al. (2007). Putative role of beta-1,3 glucans in Candida albicans biofilm resistance. Antimicrob. Agents Chemother. 51, 510-520. doi: 10.1128/AAC.01056-06

Nobre, L. S., Todorovic, S., Tavares, A. F., Oldfield, E., Hildebrandt, P., Teixeira, M., et al. (2010). Binding of azole antibiotics to Staphylococcus aureus flavohemoglobin increases intracellular oxidative stress. J. Bacteriol. 192, 15271533. doi: 10.1128/JB.01378-09

O’Donnell, L. E., Smith, K., Williams, C., Nile, C. J., Lappin, D. F., Bradshaw, D., et al. (2016). Dentures are a reservoir for respiratory pathogens. J. Prosthodont. 25, 99-104. doi: 10.1111/jopr.12342

Peleg, A. Y., Hogan, D. A., and Mylonakis, E. (2010). Medically important bacterial-fungal interactions. Nat. Rev. Microbiol. 8, 340-349. doi: 10.1038/ nrmicro2313

Peters, B. M., Jabra-Rizk, M. A., O’may, G. A., Costerton, J. W., and Shirtliff, M. E. (2012a). Polymicrobial interactions: impact on pathogenesis and human disease. Clin. Microbiol. Rev. 25, 193-213. doi: 10.1128/CMR.00013-11 
Peters, B. M., Jabra-Rizk, M. A., Scheper, M. A., Leid, J. G., Costerton, J. W., and Shirtliff, M. E. (2010). Microbial interactions and differential protein expression in Staphylococcus aureus -Candida albicans dual-species biofilms. FEMS Immunol. Med. Microbiol. 59, 493-503. doi: 10.1111/j.1574-695X.2010. 00710.x

Peters, B. M., and Noverr, M. C. (2013). Candida albicans-Staphylococcus aureus polymicrobial peritonitis modulates host innate immunity. Infect. Immun. 81, 2178-2189. doi: 10.1128/IAI.00265-13

Peters, B. M., Ovchinnikova, E. S., Krom, B. P., Schlecht, L. M., Zhou, H., Hoyer, L. L., et al. (2012b). Staphylococcus aureus adherence to Candida albicans hyphae is mediated by the hyphal adhesin Als3p. Microbiology 158, 2975-2986. doi: 10.1099/mic.0.062109-0

Pierce, C. G., Uppuluri, P., Tristan, A. R., Wormley, FL Jr., Mowat, E., Ramage, G., et al. (2008). A simple and reproducible 96-well plate-based method for the formation of fungal biofilms and its application to antifungal susceptibility testing. Nat. Protoc. 3, 1494-1500. doi: 10.1038/nport.2008.141

Rajendran, R., Borghi, E., Falleni, M., Perdoni, F., Tosi, D., Lappin, D. F., et al. (2015). Acetylcholine protects against Candida albicans infection by inhibiting biofilm formation and promoting hemocyte function in a Galleria mellonella infection model. Eukaryot. Cell 14, 834-844. doi: 10.1128/EC.00067-15

Rajendran, R., May, A., Sherry, L., Kean, R., Williams, C., Jones, B. L., et al. (2016a). Integrating Candida albicans metabolism with biofilm heterogeneity by transcriptome mapping. Sci. Rep. 6:35436. doi: 10.1038/srep35436

Rajendran, R., Sherry, L., Deshpande, A., Johnson, E. M., Hanson, M. F., Williams, C., et al. (2016b). A prospective surveillance study of candidaemia: epidemiology, risk factors, antifungal treatment and outcome in hospitalized patients. Front. Microbiol. 7:915. doi: 10.3389/fmicb.2016.00915

Rajendran, R., Sherry, L., Lappin, D. F., Nile, C. J., Smith, K., Williams, C., et al. (2014). Extracellular DNA release confers heterogeneity in Candida albicans biofilm formation. BMC Microbiol. 14:303. doi: 10.1186/s12866-014-0303-6

Ramage, G., Vande Walle, K., Wickes, B. L., and Lopez-Ribot, J. L. (2001). Standardized method for in vitro antifungal susceptibility testing of Candida albicans biofilms. Antimicrob. Agents Chemother. 45, 2475-2479. doi: 10.1128/ AAC.45.9.2475-2479.2001

Sancho, S., Artero, A., Zaragoza, R., Camarena, J. J., Gonzalez, R., and Nogueira, J. M. (2012). Impact of nosocomial polymicrobial bloodstream infections on the outcome in critically ill patients. Eur. J. Clin. Microbiol. Infect. Dis. 31, 1791-1796. doi: 10.1007/s10096-011-1503-8

Sapaar, B., Nur, A., Hirota, K., Yumoto, H., Murakami, K., Amoh, T., et al. (2014). Effects of extracellular DNA from Candida albicans and pneumonia-related pathogens on Candida biofilm formation and hyphal transformation. J. Appl. Microbiol. 116, 1531-1542. doi: 10.1111/jam.12483

Schlecht, L. M., Peters, B. M., Krom, B. P., Freiberg, J. A., Hansch, G. M., Filler, S. G., et al. (2015). Systemic Staphylococcus aureus infection mediated by Candida albicans hyphal invasion of mucosal tissue. Microbiology 161, 168-181. doi: 10.1099/mic.0.083485-0

Sheppard, D. C., Yeaman, M. R., Welch, W. H., Phan, Q. T., Fu, Y., Ibrahim, A. S., et al. (2004). Functional and structural diversity in the Als protein family of Candida albicans. J. Biol. Chem. 279, 30480-30489. doi: 10.1074/jbc. M401929200

Sherry, L., Lappin, G., O’donnell, L. E., Millhouse, E., Millington, O. R., Bradshaw, D. J., et al. (2016). Viable compositional analysis of an eleven species oral polymicrobial biofilm. Front. Microbiol. 7:912. doi: 10.3389/fmicb.2016.00912
Sherry, L., Rajendran, R., Lappin, D. F., Borghi, E., Perdoni, F., Falleni, M., et al. (2014). Biofilms formed by Candida albicans bloodstream isolates display phenotypic and transcriptional heterogeneity that are associated with resistance and pathogenicity. BMC Microbiol. 14:182. doi: 10.1186/1471-2180-14-182

Shine, W. E., Silvany, R., and Mcculley, J. P. (1993). Relation of cholesterolstimulated Staphylococcus aureus growth to chronic blepharitis. Invest. Ophthalmol. Vis. Sci. 34, 2291-2296.

Smith, K., Perez, A., Ramage, G., Lappin, D., Gemmell, C. G., and Lang, S. (2008). Biofilm formation by Scottish clinical isolates of Staphylococcus aureus. J. Med. Microbiol. 57, 1018-1023. doi: 10.1099/jmm.0.2008/000968-0

Spellberg, B., Ibrahim, A. S., Yeaman, M. R., Lin, L., Fu, Y., Avanesian, V., et al. (2008). The antifungal vaccine derived from the recombinant $\mathrm{N}$ terminus of Als3p protects mice against the bacterium Staphylococcus aureus. Infect. Immun. 76, 4574-4580. doi: 10.1128/IAI.00700-08

Townsend, E., Sherry, L., Rajendran, R., Hansom, D., Butcher, J., Mackay, W. G., et al. (2016). Development and characterisation of a novel three-dimensional interkingdom wound biofilm model. Biofouling 32, 1259-1270. doi: 10.1080/ 08927014.2016.1252337

Unnanuntana, A., Bonsignore, L., Shirtliff, M. E., and Greenfield, E. M. (2009). The effects of farnesol on Staphylococcus aureus biofilms and osteoblasts. An in vitro study. J. Bone Joint Surg. Am. 91, 2683-2692. doi: 10.2106/JBJS.H. 01699

Valenza, G., Tappe, D., Turnwald, D., Frosch, M., Konig, C., Hebestreit, H., et al. (2008). Prevalence and antimicrobial susceptibility of microorganisms isolated from sputa of patients with cystic fibrosis. J. Cyst. Fibros. 7, 123-127. doi: 10.1016/j.jcf.2007.06.006

Vanden Bossche, H., Marichal, P., Willemsens, G., Bellens, D., Gorrens, J., Roels, I., et al. (1990). Saperconazole: a selective inhibitor of the cytochrome P-450dependent ergosterol synthesis in Candida albicans, Aspergillus fumigatus and Trichophyton mentagrophytes. Mycoses 33, 335-352.

Vandenbosch, D., Braeckmans, K., Nelis, H. J., and Coenye, T. (2010). Fungicidal activity of miconazole against Candida spp. biofilms. J. Antimicrob. Chemother. 65, 694-700. doi: 10.1093/jac/dkq019

Weidj, S., Haggarty, J., Kean, R., Cojocariu, C. I., Silcock, P. J., Rajendran, R., et al. (2016). A novel targeted/untargeted GC-Orbitrap metabolomics methodology applied to Candida albicans and Staphylococcus aureus biofilms. Metabolomics 12, 189. doi: 10.1007/s11306-016-1134-2

Wisplinghoff, H., Bischoff, T., Tallent, S. M., Seifert, H., Wenzel, R. P., and Edmond, M. B. (2004). Nosocomial bloodstream infections in US hospitals: analysis of 24,179 cases from a prospective nationwide surveillance study. Clin. Infect. Dis. 39, 309-317. doi: $10.1086 / 421946$

Conflict of Interest Statement: The authors declare that the research was conducted in the absence of any commercial or financial relationships that could be construed as a potential conflict of interest.

Copyright (c) 2017 Kean, Rajendran, Haggarty, Townsend, Short, Burgess, Lang, Millington, Mackay, Williams and Ramage. This is an open-access article distributed under the terms of the Creative Commons Attribution License (CC BY). The use, distribution or reproduction in other forums is permitted, provided the original author(s) or licensor are credited and that the original publication in this journal is cited, in accordance with accepted academic practice. No use, distribution or reproduction is permitted which does not comply with these terms. 